\title{
Sistema modular de telemetría para medición de parámetros en el hogar, basado en el internet de las cosas
}

\section{Modular telemetry system to measure parameters in home, based on internet of things}

HERNÁNDEZ-AGUIRRE, Fredy Alberto†*, CHÁVEZ, José Manuel, ESPEJEL-BLANCO Daniel Fernando y YANEZ-PRECIADO, Jorge Alberto

División de Estudio de Posgrado e Investigación, Tecnológico Nacional de México/Instituto Tecnológico de Hermosillo

ID $1^{\mathrm{er}}$ Autor: Fredy Alberto, Hernández-Aguirre / ORC ID: 0000-0001-9208-5299, CVU CONACYT ID: 611800

ID $1^{\mathrm{er}}$ Coautor: José Manuel, Chávez / ORC ID: 0000-0001-9708-5925, CVU CONACYT ID: 806766

ID $2^{\text {do }}$ Coautor: Daniel Fernando, Espejel-Blanco / ORC ID: 0000-0002-9692-013X, CVU CONACYT ID: 304845

ID $3^{\text {er }}$ Coautor: Jorge Alberto, Yanez-Preciado / ORC ID: 0000-0002-6150-0119, CVU CONACYT ID: 1005402

DOI: $10.35429 /$ JOCT.2020.14.4.17.27

Recibido 16 de Septiembre, 2020; Aceptado 30 Diciembre, 2020

\begin{abstract}
Resumen
El objetivo principal del presente artículo es dar a conocer el desarrollo y los resultados obtenidos en el diseño de un sistema modular de telemetría para la medición de distintos parámetros en el hogar y tener la posibilidad de conocer y monitorear los datos de manera remota a través de una aplicación móvil. Haciendo uso de instrumentación se puede realizar la medición de la energía eléctrica consumida en el hogar ( $\mathrm{kWh}$ ), la cantidad de agua consumida, presencia de gas LP, iluminación interior, temperatura y la energía generada por un panel fotovoltaico interconectado a la red eléctrica. La arquitectura propuesta para el desarrollo de esta aplicación está basada en el modelo de tres capas del IoT (Internet of Things). Se utiliza el módulo NodeMCU®, basado en el chip ESP8266, para la lectura de los parámetros propuestos anteriormente, estos datos son transmitidos por medio de un protocolo de comunicación Wi-Fi (Wireless Fidelity), denominado Websockets ${ }^{\circledR}$, y posteriormente son enviados a una base de datos utilizando el servicio de Firebase ${ }^{\circledR}$, comunicados con una aplicación móvil para su visualización en una interfaz gráfica programada en Android Studio®. El usuario podrá monitorear los distintos parámetros en tiempo real y con base a su criterio, puede ejercer acciones preventivas y correctivas para el aprovechamiento consiente de los recursos energéticos.
\end{abstract}

Telemetría, Internet de las cosas, Instrumentación, fotovoltaico

\begin{abstract}
In this article, we discuss the development and results obtained in the design of a modular telemetry system for measurement of different parameters at home and having the possibility of knowing and monitoring the data remotely through a mobile application. Using instrumentation, it is planned to measure electrical energy consumed at home in $\mathrm{kWh}$, the amount of water consumed, pressure and presence of LP gas, interior lighting, temperature and the energy generated by a photovoltaic panel interconnected to the electrical grid. The architecture proposed for the development of this application is based on the three-layer model of the IoT (Internet of Things), making use of a microcontroller based on the ESP8266 chip called NodeMCUß, the reading of the parameters proposed above is performed, and these data are transmitted through a Wi-Fi (Wireless Fidelity) communication protocol called Websockets $®$, the data is sent to a database on the internet using Firebase ${ }^{\circledR}$ service, later this database communicates with a mobile application in which displays the data collected by the microcontroller in a graphical interface programmed with Android Studio®. The user will be able to monitor the different parameters in real-time and based on his criteria, take preventive and corrective actions for the conscious use of his energy resources.
\end{abstract}

Telemetry, Mobile application, Internet of things, Instrumentation

Citación: HERNÁNDEZ-AGUIRRE, Fredy Alberto, CHÁVEZ, José Manuel, ESPEJEL-BLANCO Daniel Fernando y YANEZ-PRECIADO, Jorge Alberto. Sistema modular de telemetría para medición de parámetros en el hogar, basado en el internet de las cosas. Revista de Tecnologías Computacionales. 2020. 4-14: 17-27

\footnotetext{
* Correspondencia del Autor (fredy.hernandeza@hermosillo.tecnm.mx)

$\dagger$ Investigador contribuyendo como primer autor.
} 


\section{Introducción}

La energía eléctrica se ha convertido en un recurso indispensable en el día a día de la sociedad, un gran porcentaje de esta energía es desperdiciada en el transporte y la distribución de la electricidad, por lo que es importante hacer uso de las tecnologías actuales para reducir significativamente la pérdida de energía [1].

Los medidores inteligentes para electricidad se están implementando desde 2011 en hogares privados por todo el mundo, principalmente por decisiones gubernamentales para cumplir objetivos de ahorro de energía. Por otro lado, los medidores inteligentes de electricidad junto con los de gas, calefacción y agua se pueden interconectar en una gran red que ofrece un valor potencial para implementar ahorro de energía y otros servicios relacionados con la energía, siempre que se implemente una interfaz con el usuario final [2].

En el artículo [3], el autor destaca la importancia de combatir el robo de electricidad implementando un AMR (Automatic Meter Reading), que además de ayudar a combatir estas malas prácticas que se efectúan en países en desarrollo, elimina la necesidad de una persona perteneciente a la empresa prestadora del servicio eléctrico tome lecturas de los medidores instalados en hogares u oficinas. El prototipo desarrollado en este artículo está basado en el microcontrolador Arduino®, con un módulo GSM para alertas y un medidor de energía eléctrica.

En el artículo [4], Vu Chien Thang propone una solución para las industrias que utilizan el recurso del agua en Vietnam, implementando un medidor de calidad y consumo del agua con un microcontrolador TIVAC TM4C123 y un módulo sim 908 para comunicar los datos a un servidor de Internet, y posteriormente pueden ser visualizados en una página de Internet, los resultados demuestran que la solución ha operado bien en las condiciones reales.

El objetivo del proyecto desarrollado es el diseño de un sistema modular de telemetría para un medidor inteligente, capaz de tomar lecturas del consumo energético $(\mathrm{kWh})$, consumo de agua (litros), presencia de gas LP, iluminación interior, temperatura y la energía eléctrica generada por un panel fotovoltaico.
El sistema es modular, lo que permite adaptarse a las diferentes arquitecturas en las instalaciones residenciales.

El reto tecnológico de este proyecto consiste en el desarrollo de un sistema modular de telemetría con un costo accesible y de fácil instalación en las residencias de México; actualmente hay ofertas norteamericanas de medidores inteligentes para el hogar de 3 parámetros, electricidad, agua y gas, a un costo superior a los \$121,00.00 MXN.

\section{Metodología}

En la figura 1, se muestran las diversas etapas metodológicas utilizadas para el desarrollo del proyecto.

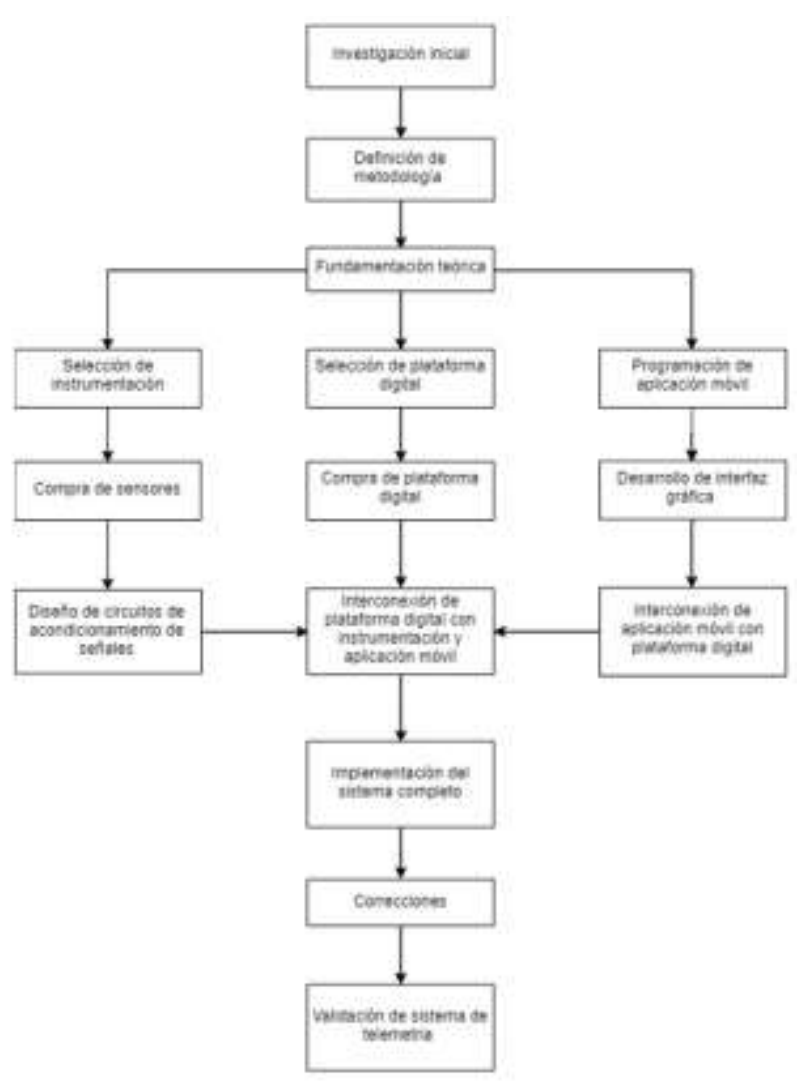

Figura 1 Metodología implementada para el desarrollo del proyecto

Fuente: Elaboración Propia

Para el desarrollo del prototipo, es fundamental realizar investigación con el fin de conocer los trabajos previos que se hayan hecho para complementar los conocimientos adquiridos y justificar el desarrollo de la ingeniería, se utilizó la metodología científica basada en el diseño de circuitos electrónicos y posteriores pruebas para su validación. 
El proyecto se puede dividir en tres etapas principales:

- Instrumentación: Selección y compra de los sensores requeridos para el proyecto, esta etapa incluye el desarrollo de los diferentes circuitos de acondicionamiento de señal requeridos.

- Interconexión: Desarrollo de algoritmos de comunicación para interconectar la instrumentación, una plataforma digital para procesamiento de datos, un servidor en Internet para el almacenamiento de datos y una aplicación móvil.

- $\quad$ Interfaz gráfica: Desarrollo de aplicación móvil para Android® donde el usuario final pueda consultar los datos recabados de los distintos sensores en tiempo real.

Para llevar a cabo con éxito las etapas mencionadas anteriormente, se requiere realizar una investigación inicial de la problemática, conocer las alternativas existentes para analizar su arquitectura y buscar posibles mejoras.

\section{Propuesta de solución}

Se realizó una investigación y comparación para seleccionar la instrumentación requerida por el sistema de telemetría modular; considerando las características de estos sensores se diseñaron los circuitos de acondicionamiento necesarios para que puedan comunicarse con la plataforma digital, encargada de recabar los distintos datos y digitalizarlos en un servidor en Internet, para que puedan ser mostrados en una aplicación móvil.

Tomando como base la arquitectura de una aplicación IoT de tres capas, a continuación, se describen las diferentes capas del prototipo:

- La capa de percepción está compuesta por los diferentes sensores requeridos para la lectura de los parámetros.

- La capa de red consiste en un microcontrolador para interpretar los datos y centralizarlos en un servidor de Internet.

- La capa de aplicación es una interfaz gráfica desarrollada para el sistema operativo Android, con la finalidad de visualizar las mediciones registradas por el sistema de telemetría.
Para el diseño del sistema de telemetría, se propuso el uso de una aplicación móvil por las ventajas que ofrece a la hora de diseñar una interfaz gráfica. El entorno de desarrollo Android Studio facilita la adaptación de distintos dispositivos móviles Android, permitiendo enfocar la programación en algoritmos de comunicación de datos y la visualización de los mismos de manera funcional.

El protocolo de comunicación utilizado para la arquitectura de este proyecto es por Websockets, es un protocolo de comunicación WiFi bidireccional basado en HTTP (Hyper Text Transport Protocol).

En la figura 2 se muestra la arquitectura utilizada en la propuesta de solución para la problemática planteada con respecto al sistema de telemetría para el monitoreo de los servicios básicos.

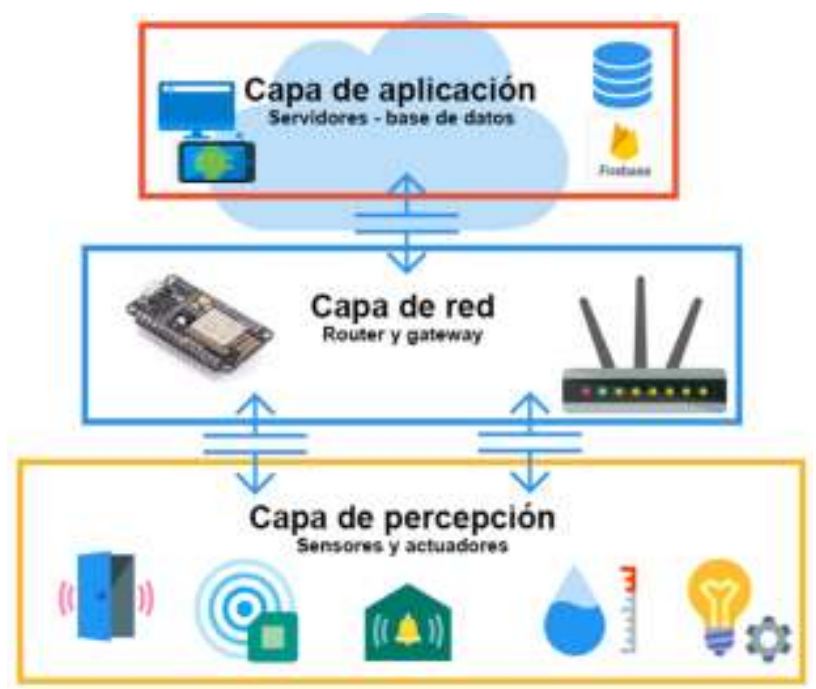

Figura 2 Arquitectura basada en el modelo de tres capas del internet de las cosas

Fuente: [5]

\section{Desarrollo del prototipo}

\subsection{Selección y caracterización de la instrumentación}

Sensor de energía eléctrica PZEM-004T. Está basado en el chip SD3004 de SDIC Microelectronics ${ }^{\circledR}$, trabaja con un microcontrolador Atmel ${ }^{\circledR}$ que cuenta con una memoria PROM de 2kbit, la cual permite hacer más de 1 millón de ciclos de lectura/escritura, y retiene los datos hasta por 200 años, este sensor es capaz de medir corrientes de 0 - 100 Amperes, voltajes de 80 - $260 \mathrm{VAC}$, para la transmisión de datos cuenta con una interfaz serial con una velocidad de 9600 baudios [6]. 
En la figura 3 se muestra el sensor de energía eléctrica y el transformador de corriente no invasivo utilizado para las mediciones eléctricas de corriente instantánea.

Para probar la funcionalidad del sensor de energía eléctrica se contempla la planta de prueba de la figura 4. Para esta prueba, se utiliza una carga de $30 \mathrm{~W}$, lectura que nos debería de indicar el sensor de energía eléctrica.

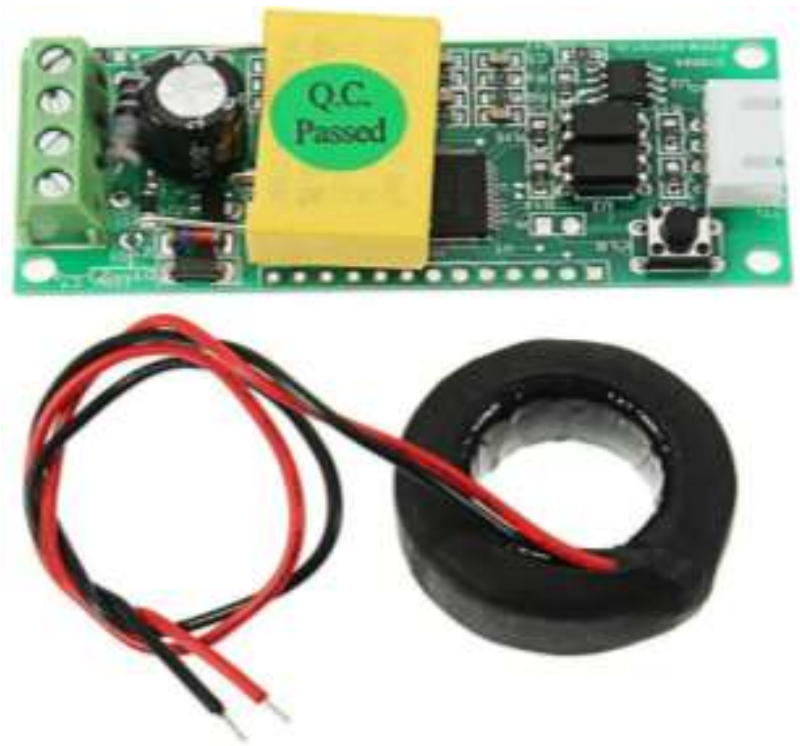

Figura 3 Sensor PZEM-004T y transformador de corriente

Fuente: [6]

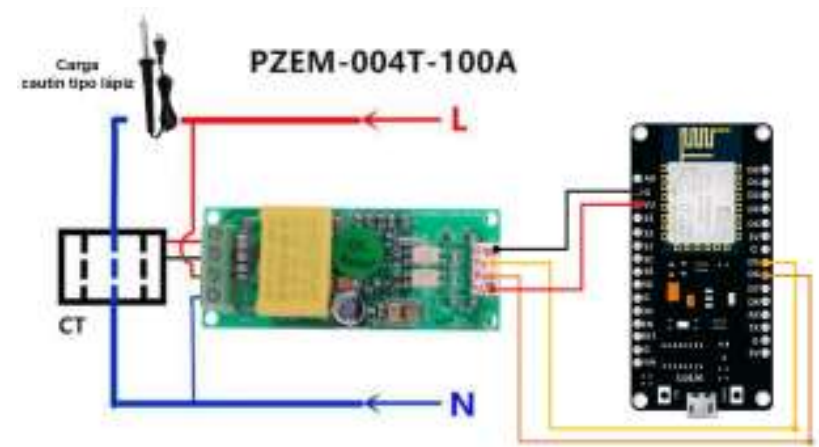

Figura 4 Diagrama de conexión para planta de prueba con el sensor PZEM-004T

Fuente: Elaboración Propia

Utilizando la ley de Watt, ecuación 1, se pueden calcular los parámetros eléctricos de la carga (cautín de 30W), [7].

$$
P=V * I
$$

Para conocer el voltaje de operación, se realiza una medición entre la terminal de línea y de neutro con un multímetro de marca Steren modelo MUL-500, figura 5.
$\mathrm{Al}$ conocer la potencia y el voltaje del circuito, se puede calcular la corriente:

$P=V * I$

$$
I=\frac{P}{V}
$$

$$
I=\frac{30 \mathrm{~W}}{122.4 \mathrm{~V}}=0.245 \mathrm{~A}
$$

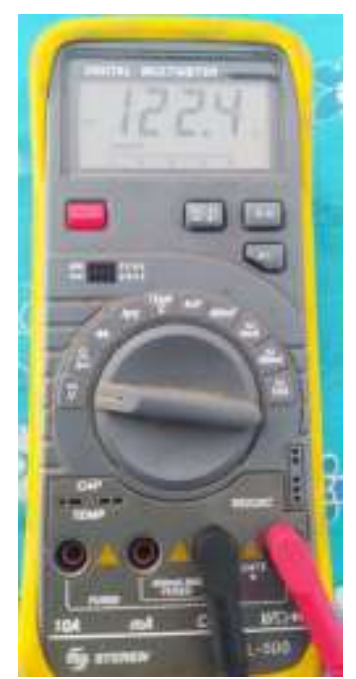

Figura 5 Lectura de voltaje en terminales línea y neutro para planta de prueba

Fuente: Elaboración Propia

En la figura 6 se muestra la conexión del sensor para medir la corriente calculada.

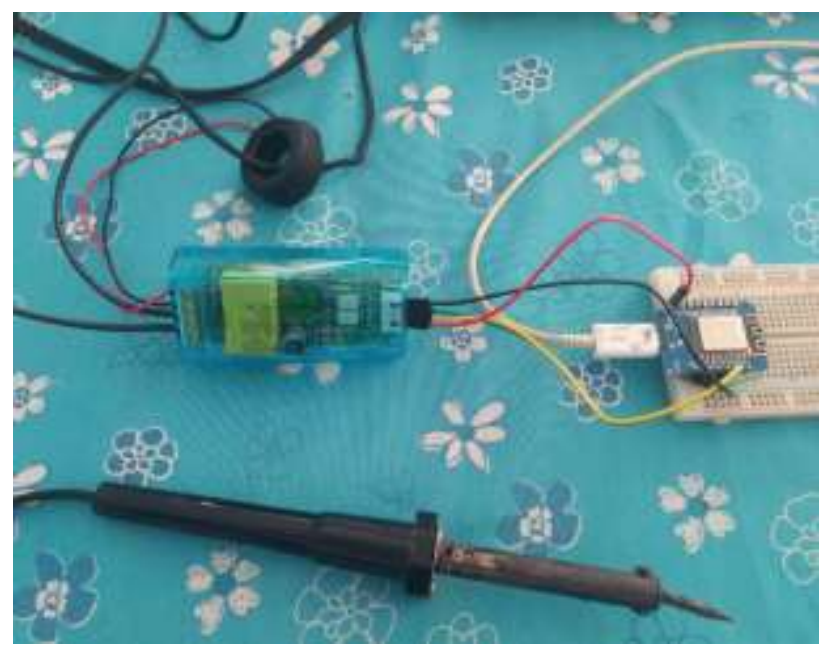

Figura 6 Planta de prueba para el sensor de energía eléctrica

Fuente: Elaboración Propia

En la figura 7 se muestran las mediciones realizadas, monitoreadas a través de comunicación serial con módulo Arduino. 


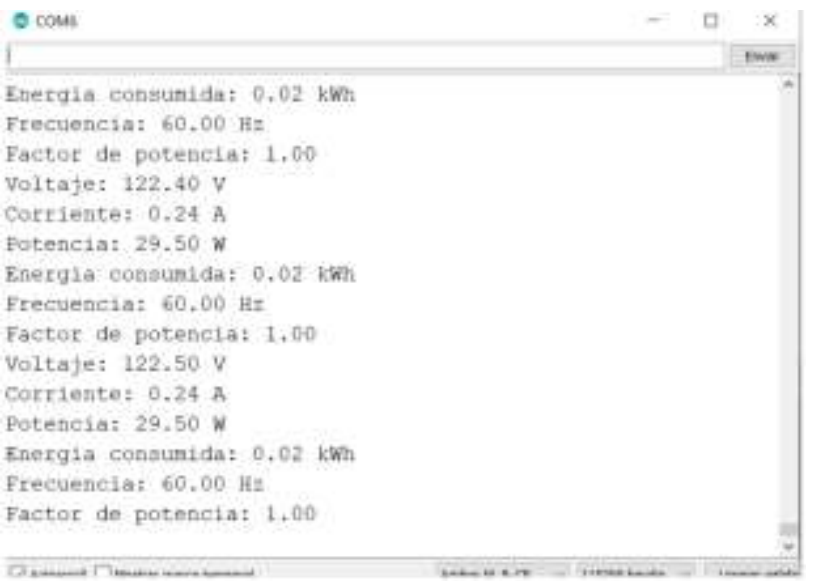

Figura 7 Monitor serial donde se muestran las lecturas del sensor de energía eléctrica

Fuente: Elaboración Propia

Comparando las mediciones realizadas con e multímetro y el sensor de corriente, se puede validar la funcionalidad del sensor PZEM004.

Sensor de flujo de agua YF-S201. Es un sensor de flujo usado en distintas aplicaciones, pues el caudal o flujo es un parámetro necesario en varios procesos, a nivel doméstico se puede utilizar para medir el consumo de agua. El sensor internamente tiene un rotor cuyas paletas tienen un imán, la cámara en donde se encuentra el rotor es totalmente aislado evitando fugas de agua, externamente a la cámara tiene un sensor de efecto hall que detecta el campo magnético del imán de las paletas y con esto el movimiento del rotor, el sensor de efecto hall envía los pulsos por uno de los cables del sensor, los pulsos son convertidos posteriormente a flujo [8]. En la figura 8 podemos observar el sensor de flujo de agua.

Este sensor tiene tres cables para su conexión, rojo y negro para la alimentación y amarillo para la salida de los pulsos, una señal cuadrada cuya frecuencia es proporcional al caudal.

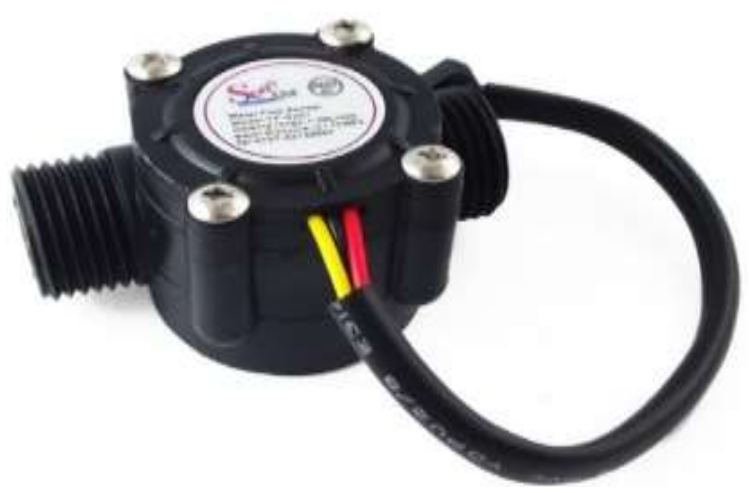

Figura 8 Sensor de flujo de agua YF-S201

Fuente: [9]

ISSN 2523-6814

ECORFAN® Todos los derechos reservado
Para la conexión del microcontrolador con el sensor se realizaron las conexiones mostradas en la figura 9.

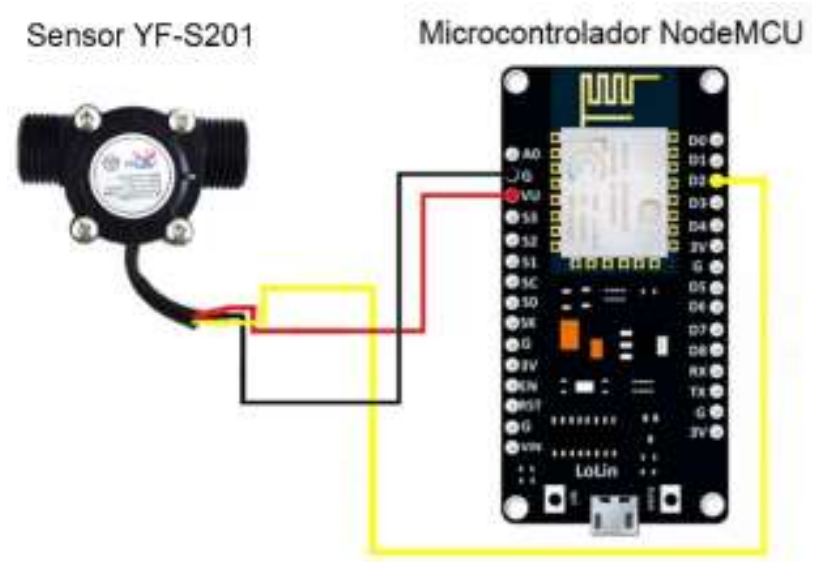

Figura 9 Diagrama de conexión de sensor YF-S201 con microcontrolador

Fuente: Elaboración Propia

Para probar el sensor se utilizó la planta de pruebas que se muestra en la figura 10 . Se cuenta con un tanque de agua conectado a una bomba de agua para aumentar la presión y mantener un flujo constante. La bomba de agua se conecta al sensor utilizando un acoplamiento reductor de cobre, y el flujo atraviesa el caudalímetro, vaciándose en un contenedor de agua de 1 litro.

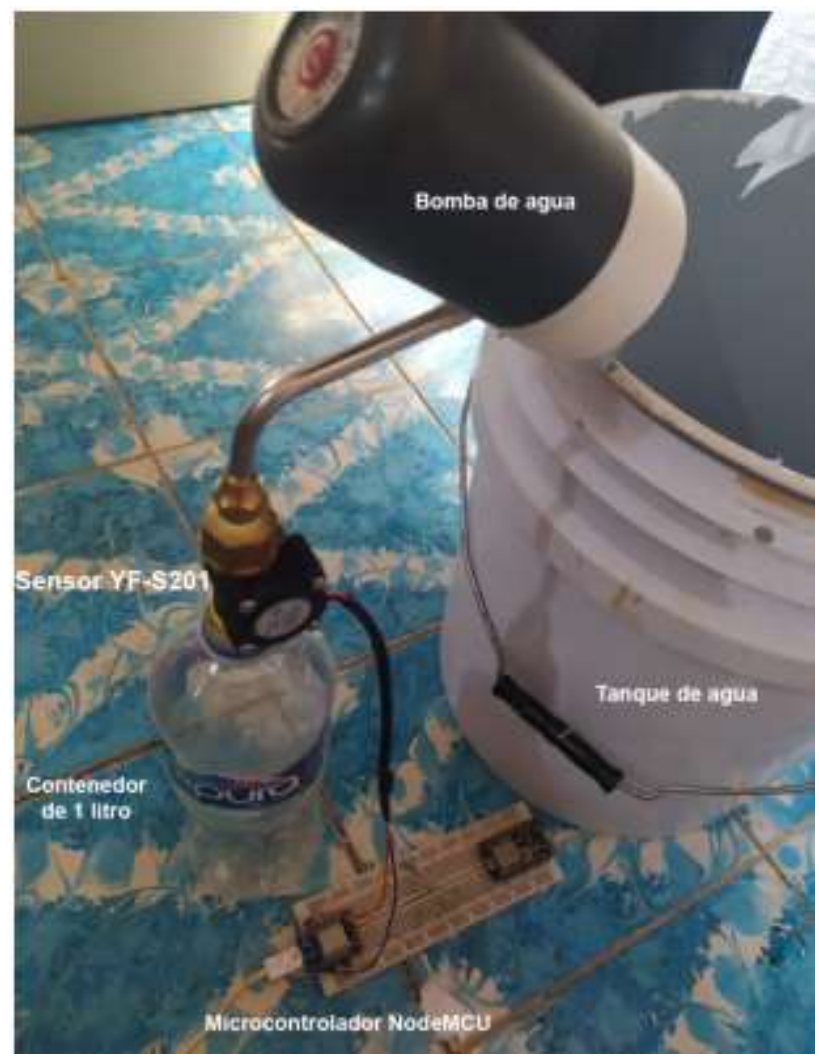

Figura 10 Planta de pruebas para sensor de agua YF-S201 Fuente: Elaboración Propia 
Para obtener mayor precisión en las lecturas del sensor fue necesario realizar una calibración, para llevar a cabo la calibración se realizó un script encargado de contar los pulsos que recibía el microcontrolador del sensor cuando se llenaba un contenedor de un litro de agua. Se realizaron 10 pruebas para obtener el promedio de los distintos valores y calcular el factor de conversión. En la tabla 1 se muestran los valores obtenidos.

Con estos 10 cálculos se puede obtener el promedio de pulsos por litro para crear un script que sea capaz de obtener el valor del consumo de agua en litros, para probarlo se hicieron 2 pruebas utilizando la misma planta cambiando únicamente el contenedor de agua, para la primera prueba se utilizó un contenedor de 600 mililitros.

\begin{tabular}{|l|l|l|}
$\begin{array}{c}\text { Número de } \\
\text { prueba }\end{array}$ & $\begin{array}{c}\text { Tamaño de } \\
\text { contenedor }\end{array}$ & \multicolumn{1}{c|}{$\begin{array}{c}\text { Número de } \\
\text { pulsos }\end{array}$} \\
\hline Prueba 1 & 1 litro & 457 \\
\hline Prueba 2 & 1 litro & 435 \\
\hline Prueba 3 & 1 litro & 425 \\
\hline Prueba 4 & 1 litro & 414 \\
\hline Prueba 5 & 1 litro & 415 \\
\hline Prueba 6 & 1 litro & 430 \\
\hline Prueba 7 & 1 litro & 420 \\
\hline Prueba 8 & 1 litro & 418 \\
\hline Prueba 9 & 1 litro & 418 \\
\hline Prueba 10 & 1 litro & 437 \\
\hline
\end{tabular}

Tabla 1 Conteo de pulsos con sensor de flujo

En la figura 11 se muestran las mediciones registradas con puerto serial, utilizando un contenedor de 0.6 litros.

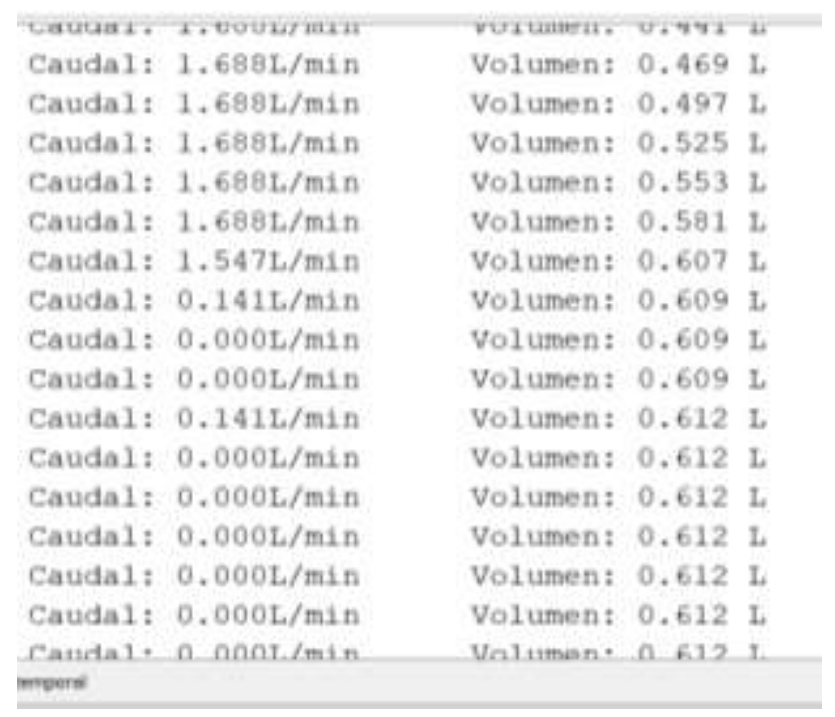

Figura 11 Monitor serial mostrando los datos de volumen de agua por el sensor de flujo de agua Fuente: Elaboración Propia
Para la segunda prueba se cambió el contenedor de agua por uno de 1.5 Litros, el microcontrolador arrojó el resultado que se observa en la figura 12.

\begin{tabular}{|c|c|c|c|c|}
\hline Caudal: & $1.688 \mathrm{~L} / \mathrm{min}$ & Volumen: & 1.118 & L \\
\hline Caudal: & $1.688 \mathrm{~L} / \mathrm{min}$ & Volumen: & 1.146 & L. \\
\hline Cauda 1: & $1.688 \mathrm{~L} / \mathrm{min}$ & Volumen: & 1.174 & $\mathrm{~L}$ \\
\hline Caudal: & $1.547 \mathrm{~L} / \mathrm{min}$ & Volumen: & 1.200 & L \\
\hline Caudal: & $1.6801 / \mathrm{min}$ & Volumen: & 1.220 & L. \\
\hline Cauda1: & $1.680 \mathrm{~L} / \mathrm{min}$ & Volumen: & 1.256 & $\mathrm{~L}$ \\
\hline CaudaI: & $1.82 \theta \mathrm{L} / \mathrm{min}$ & Volumen: & 1.287 & L \\
\hline Cauda1: & $1.688 \mathrm{~L} / \mathrm{min}$ & volumen: & 1.315 & L \\
\hline Caudal: & $1.688 \mathrm{~L} / \mathrm{min}$ & Volumen: & 1.343 & L \\
\hline Caudal: & $1.547 \mathrm{~L} / \mathrm{min}$ & Volumen: & 1.369 & $\mathrm{t}$ \\
\hline Cauda 1: & $1.547 \mathrm{~L} / \mathrm{min}$ & Volumen: & 1.395 & t \\
\hline Caudal: & 1. $68 \mathrm{eb} / \mathrm{min}$ & Volumen: & 1.423 & L \\
\hline Cauda1: & $1.547 \mathrm{~L} / \mathrm{min}$ & Volumen: & 1.449 & L. \\
\hline Cauda 1: & $1.547 \mathrm{~L} / \mathrm{min}$ & Volumen: & 1.474 & L. \\
\hline Caudal: & $1.547 \mathrm{~L} / \mathrm{min}$ & Volumen: & 1.500 & L \\
\hline Cauda1: & $1.547 \mathrm{~L} / \mathrm{min}$ & Volumen: & 1.526 & L. \\
\hline
\end{tabular}

Figura 12 Segunda prueba de sensor de flujo de agua Fuente: Elaboración Propia

Sensor de presencia de gas LP MQ-2. Este sensor es electroquímico y varia su resistencia cuando se expone a determinados gases, internamente cuenta con un calentador encargado de aumentar la temperatura interna y con esto el sensor pueda reaccionar con los gases provocando un cambio en el valor de la resistencia.

En el mercado, los sensores MQ se encuentran en módulos, lo que nos simplifica la parte de conexiones y facilitan su uso, solo basta con alimentar el módulo y empezar a leer el sensor. Estos módulos tienen una salida analógica y una salida digital, la cual internamente trabaja con un comparador y con la ayuda de un potenciómetro se puede calibrar el umbral para interpretar la salida digital como presencia o ausencia del gas.

La diferencia entre los distintos tipos de sensores MQ es la sensibilidad a cierta gama de gases, más sensibles a algunos gases que a otros, pero siempre detectan a más de un gas, por lo que es necesario revisar las hojas de datos para seleccionar el sensor adecuado para una aplicación en específico. En este caso se plantea utilizar el sensor MQ2 pues es adecuado para detectar GLP, propano, metano, alcohol, hidrógeno, humo. Siendo más sensible al GLP y propano [10]. 
Para realizar la conexión del sensor de gas con el microcontrolador es necesario utilizar el pin de entrada analógica, un pin de entrada digital, alimentación de voltaje y tierra. Figura 13.

\section{Microcontrolador NodeMCU}

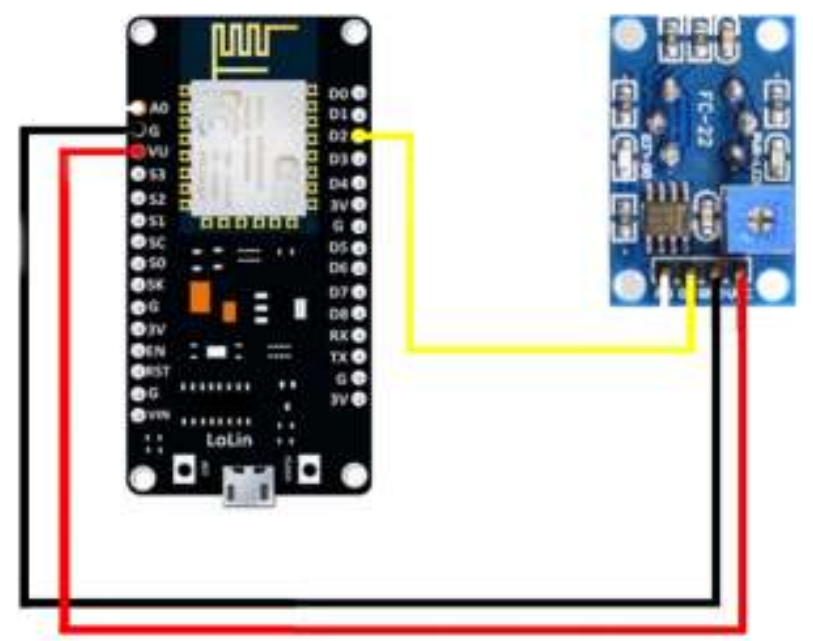

Figura 13 Diagrama de conexiones del sensor MQ-2 con el microcontrolador

Fuente: Elaboración Propia

Para la primera prueba con el sensor de gas LP, se escribió un script encargado únicamente de leer el valor de la entrada digital D2. Un nivel lógico alto en este pin, indica que no se está detectando presencia de gas, y un nivel bajo representa la detección de gas. Ajustando el potenciómetro se puede modificar el umbral de detección. En la figura 14 se muestra el circuito utilizado para las pruebas con el sensor detector de gas LP, y en la figura 15 se presentan los resultados obtenidos con monitor serial de Arduino.

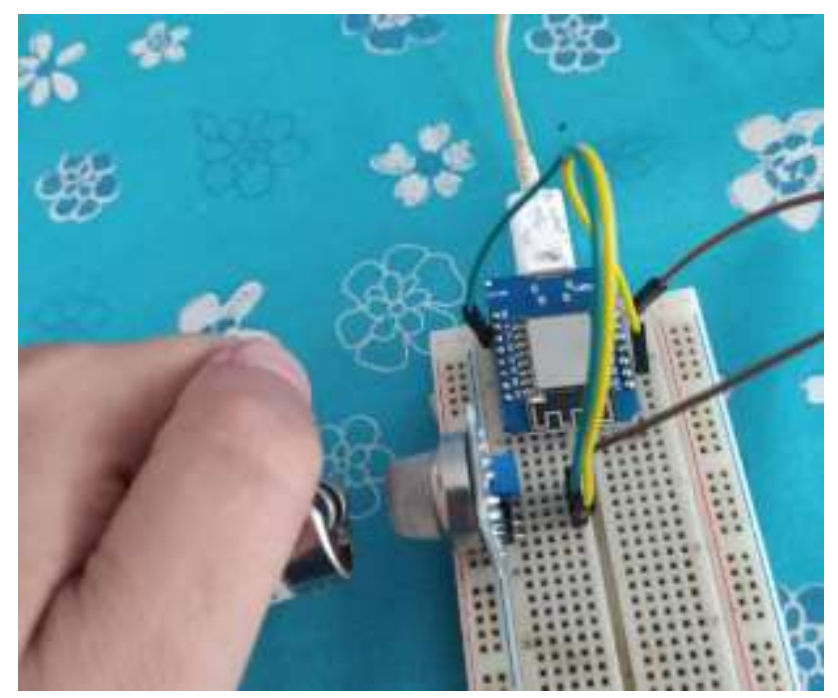

Figura 14 Prueba de detección de gas LP con sensor MQ2

Fuente: Elaboración Propia

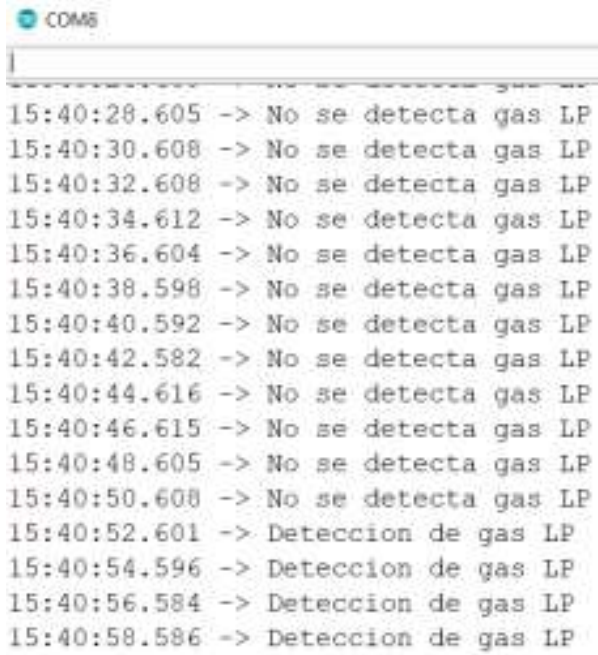

Figura 15 Monitor serial notificando la presencia de gas LP con el sensor MQ-2

Fuente: Elaboración Propia

Sensor de temperatura DS18B20. Es un sensor digital de temperatura que utiliza el protocolo 1-Wire para comunicarse. Este protocolo necesita solo un pin de datos para comunicarse y permite conectar más de un sensor en el mismo bus.

El sensor DS18B20 es fabricado por Maxim Integrated, el encapsulado de fábrica es tipo TO-92 similar al empleado en transistores pequeños. La presentación comercial más utilizada por conveniencia y robustez es la del sensor dentro de un tubo de acero inoxidable resistente al agua [11]. En la figura 16 se observa el sensor de temperatura DS18B20.

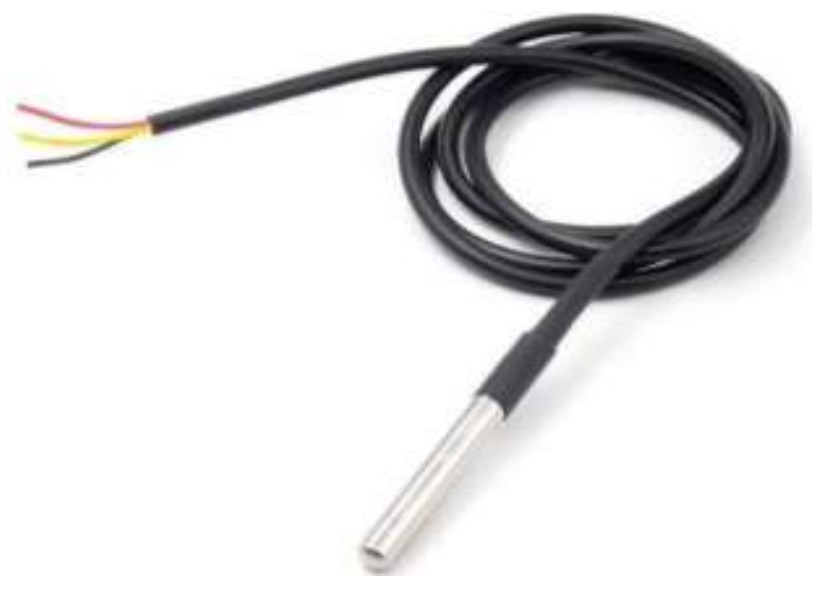

Figura 16 Sensor de temperatura DS18B20 Fuente: [12]

La conexión de este sensor es muy sencilla, únicamente se conecta el pin de color amarillo a cualquier entrada digital del microcontrolador NodeMCU (pin digital 2). 
En la figura 17 se observa la conexión del sensor con el microcontrolador.

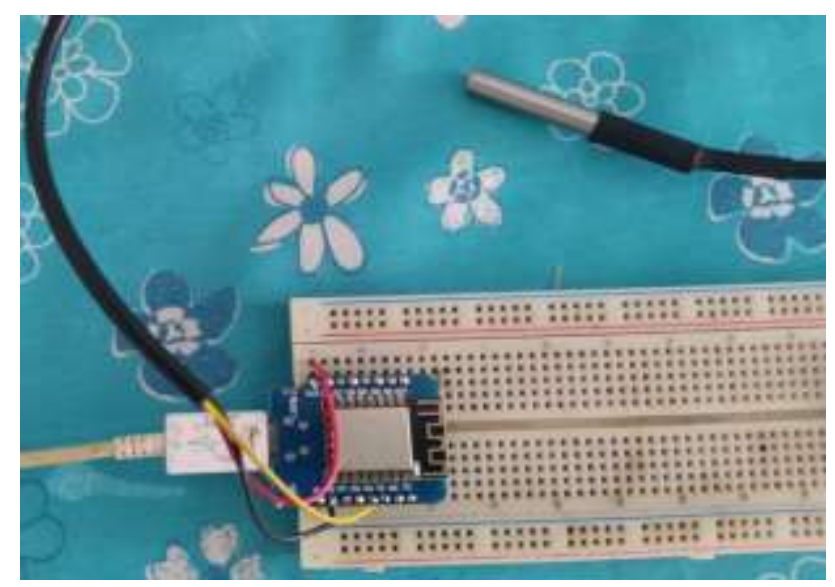

Figura 17 Conexión del microcontrolador con el sensor de temperatura DS18B20

Fuente: Elaboración Propia

Con esta conexión se puede programar un script capaz de hacer lecturas de temperatura, en la figura 18 se adjunta la captura de pantalla del monitor serial del programa de Arduino, en donde se observa la lectura del sensor de temperatura DS18B20, se toma una lectura cada segundo ya que así fue programado el script para esta prueba.

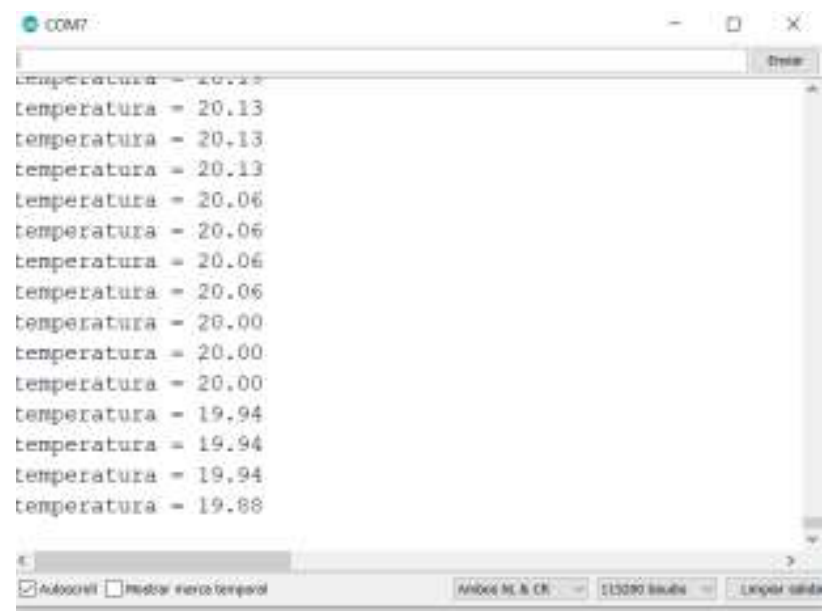

Figura 18 Monitor serial donde se muestra las lecturas de temperatura del sensor DS18B2

Fuente: Elaboración Propia

Sensor de iluminación BH1750. Se trata de un sensor de luz, que a diferencia del LDR (Light Dependent Resistor) convencional, este es digital y entrega valores de medición en Lux (lumen $/ \mathrm{m}^{2}$ ) que es una unidad de medida estándar para el nivel de iluminación. Tiene alta precisión y un rango configurable entre $1 \mathrm{y}$ 65535 1x. La interfaz de comunicación es I2C, pudiéndolo implementar en la mayoría de microcontroladores [13].
Para la conexión del sensor con el microcontrolador se utiliza el diagrama que se muestra en la figura 19.

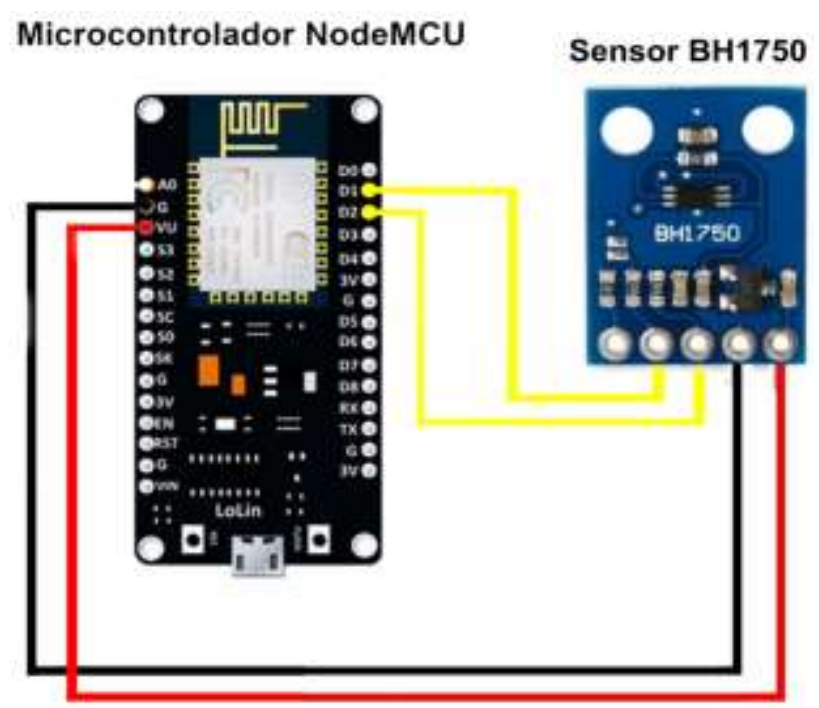

Figura 19 Diagrama de conexión del sensor de iluminación BH1750 con el microcontrolador Fuente: Elaboración Propia

En la figura 20 se muestra una foto de la conexión física del sensor de iluminación con el microcontrolador.

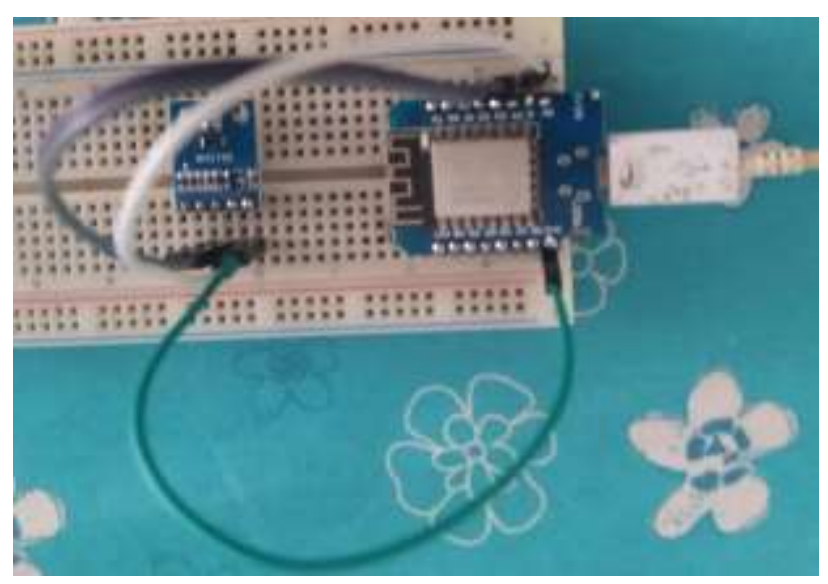

Figura 20 Conexión de sensor BH1750 con microcontrolador

Fuente: Elaboración Propia

El script programado se encarga de imprimir en el monitor serial el valor de la iluminación en Lux. En la figura 21 se adjunta una captura de pantalla donde se muestra el monitor serial imprimiendo los valores del sensor. 


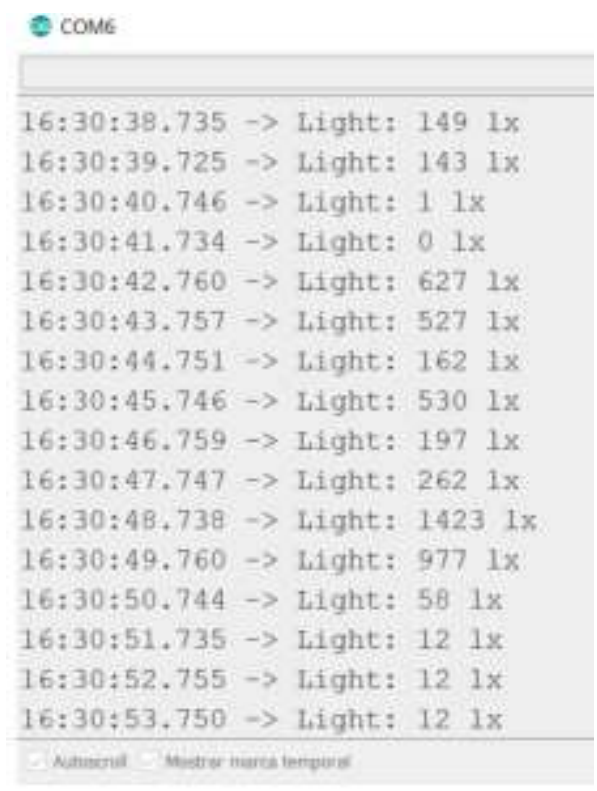

Figura 21 Monitor serial mostrando los datos del sensor de iluminación

Fuente: Elaboración Propia

\subsection{Integración de prototipo}

Una vez validados lo sensores utilizados para la medición de todos los parámetros del sistema de telemetría: sensor de corriente, de flujo de agua, detección de gas LP, temperatura e intensidad luminosa, se procede a la integración de todo el sistema de medición. Para esto se utiliza una placa fenólica de cobre perforada, que permite realizar las conexiones de los sensores con el microcontrolador. En la figura 22 se muestra el diagrama de integración de todos los sensores con el módulo NodeMCU ESP8266.

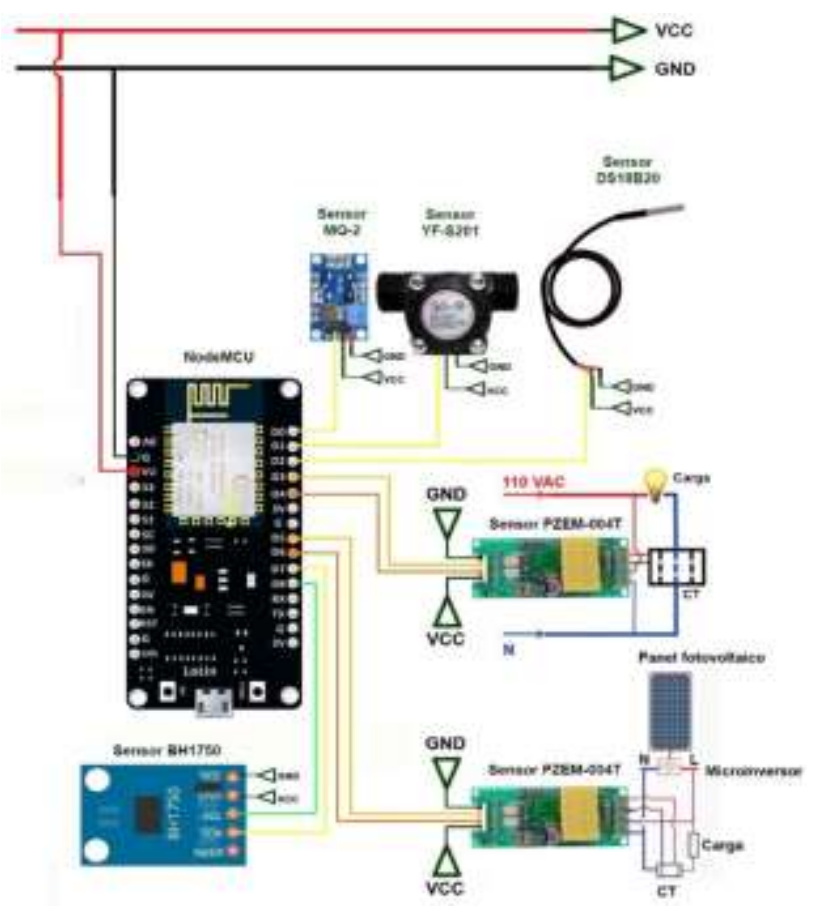

Figura 22 Diagrama de conexiones del microcontrolador con los sensores seleccionados para el proyecto

Fuente: Elaboración Propia

\subsection{Programación de Script para los sensores}

El script que desarrollado para este prototipo es ser capaz de poder conectarse a una red $\mathrm{Wi}-\mathrm{Fi}$, posteriormente conectar el microcontrolador con la base de datos de Firebase para poder cargar los datos que se obtengan de los sensores. Gracias a las pruebas individuales que se hicieron a los sensores se puede integrar de manera individual cada uno de los scripts utilizados para conformar uno solo, que a través de funciones que se ejecuten en orden, sea capaz de obtener la lectura de todos los sensores periódicamente. En la figura 23 se observa el diagrama de flujo del script utilizado para realizar la programación, este script funciona tanto para el microcontrolador NodeMCU como para el microcontrolador WEMOS D1 Mini®.

\subsection{Programación de aplicación móvil}

El desarrollo de la aplicación móvil fue realizado en Android Studio, la interfaz gráfica de la aplicación es sencilla, además de esto, la documentación que hay en Internet para conectar la base de datos de Firebase con Android Studio es muy extensa, por lo que realizar esta conexión no fue nada complicado, se relacionó un cuadro de texto con cada uno de los valores almacenados en la base de datos de los distintos parámetros, de esta manera al actualizarse en la base de datos, la aplicación móvil se actualiza de igual manera en tiempo real. 


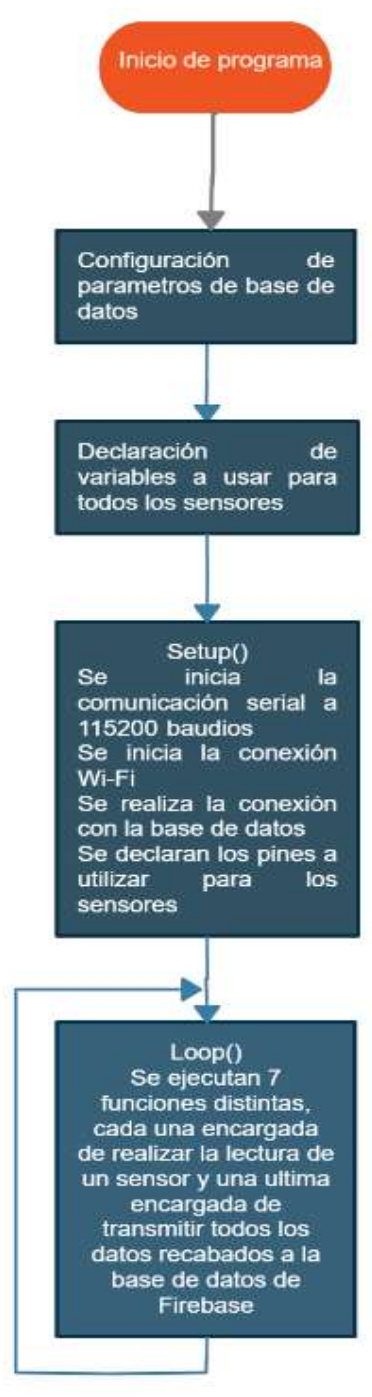

Figura 23 Diagrama de flujo de script para prototipo Fuente: Elaboración Propia

\section{Resultados}

Una vez realizada la programación de la interfaz gráfica en la aplicación móvil y del script para el microcontrolador que se encarga de recolectar los datos de los sensores, se probó el circuito unificado del microcontrolador con los sensores, figura 24.

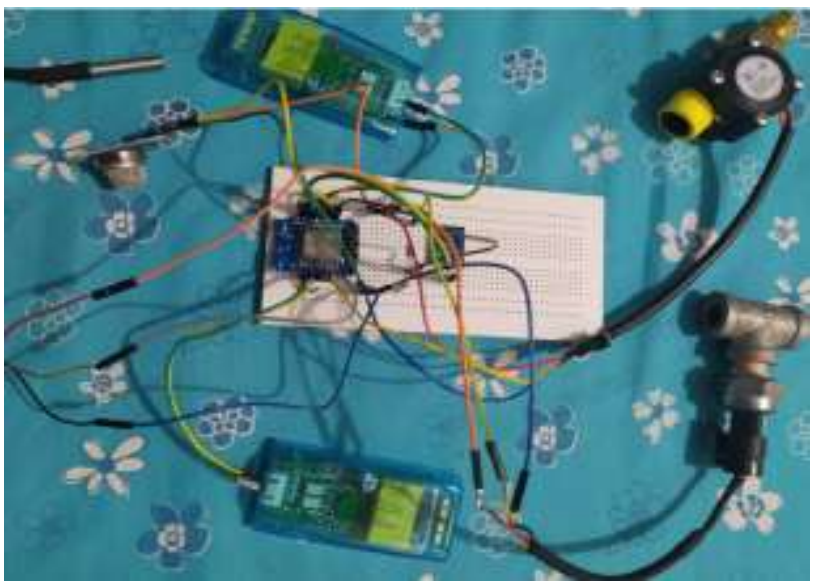

Figura 24 Circuito de microcontrolador con los sensores integrados

Fuente: Elaboración Propia

ISSN 2523-6814

ECORFAN® Todos los derechos reservado
Una vez integrados los sensores se probó cada uno simultáneamente con las plantas anteriormente comentadas, en la figura 25 se puede observar la pantalla programada de la aplicación móvil encargada de mostrar los datos en tiempo real recabados por el microcontrolador.
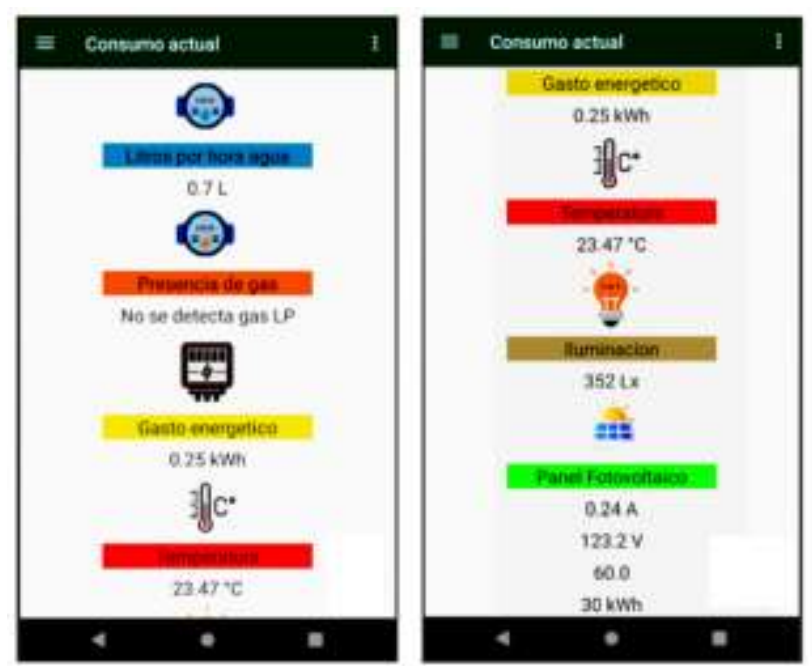

Figura 25 Pantalla para visualización de datos en tiempo real de los datos cargados por el microcontrolador a Firebase

Fuente: Elaboración Propia

\section{Conclusiones y recomendaciones}

Con el paso del tiempo se ha vuelto imprescindible el uso de las nuevas tecnologías de la información en nuestra vida diaria, es creciente la necesidad de tener información disponible en un instante preciso de tiempo. Con los resultados de la validación de las pruebas realizadas con la integración de los sensores en el sistema de telemetría propuesto, se concluye que es posible desarrollar un sistema de telemetría modular para el hogar capaz de registrar periódicamente la lectura de distintos parámetros, y además con la posibilidad de visualizar estos datos sin costo extra, gracias al desarrollo de una aplicación móvil utilizando Android Studio, todo a un precio inferior a $\$ 1,600.00$ MXN. Se recomienda continuar trabajando en la actualización de la aplicación móvil, ya que el entorno de desarrollo nos brinda capacidades muy bastas a la hora del desarrollo de algoritmos. Se pueden implementar funciones para el análisis de datos, que ayuden al usuario en la toma de decisiones con respecto al mantenimiento de infraestructura y equipos, también se pueden mostrar los costos por consumos energéticos para alertar al usuario sobre el uso inadecuado de las instalaciones. 


\section{Referencias}

[1] K. C. Chen, P. C. Yeh, H. Y. Hsieh, and S. C. Chang, "Communication infrastructure of smart grid," Final Progr. Abstr. B. - 4th Int. Symp. Commun. Control. Signal Process. ISCCSP 2010, no. March, pp. 3-5, 2010, doi: 10.1109/ISCCSP.2010.5463330.

[2] F. Benzi, N. Anglani, E. Bassi, and L. Frosini, "Electricity smart meters interfacing the households," IEEE Trans. Ind. Electron., vol. 58, no. 10, pp. 4487-4494, 2011, doi: 10.1109/TIE.2011.2107713.

[3] D. B. Gadgay, "Smart Energy Meter using Arduino UNO," Int. J. Res. Appl. Sci. Eng. Technol., vol. 7, no. 10, pp. 74-77, 2019, doi: 10.22214/ijraset.2019.10013.

[4] V. C. Thang, "A Solution for Water Factories in Vietnam using Automatic Meter Reading Technology," Int. J. Comput. Netw. Inf. Secur., vol. 10, no. 8, pp. 44-50, 2018, doi: 10.5815/ijcnis.2018.08.05.

[5] M. Jabraeil Jamali, B. Bahrami, A. Heidari, P. Allahverdizadeh, and F. Norouzi, Towards the Internet of Things: Architectures, Security, and Applications. 2019.

[6] "PZEM-004T | Specification | Price | Arduino Library | Pinout." [Online]. Available: https://innovatorsguru.com/ac-digitalmultifunction-meter-using-pzem-004t/.

[Accessed: 14-Dec-2020].

[7] M. Brand and B. Z. Rojas, "Analisis de circuitos electricos 1 (curso didactico sistematizado) ivan fernando," vol. 1, 1991.

[8] "Tutorial sensor de flujo de agua." [Online]. Available:

https://www.naylampmechatronics.com/blog/47 _tutorial-sensor-de-flujo-de-agua.html.

[Accessed: 14-Dec-2020].

[9] "Sensor de flujo de agua (Hall) 1/2" YFS201." [Online]. Available: https://www.naylampmechatronics.com/sensore s-liquido/108-sensor-de-flujo-de-agua-12-yfs201.html. [Accessed: 14-Dec-2020].
[10] "Tutorial sensores de gas MQ2, MQ3, MQ7 y MQ135." [Online]. Available: https://www.naylampmechatronics.com/blog/42 _Tutorial-sensores-de-gas-MQ2-MQ3-MQ7-yMQ13.html. [Accessed: 14-Dec-2020].

[11] "Tutorial sensor digital de temperatura DS18B20." [Online]. Available: https://www.naylampmechatronics.com/blog/46 _Tutorial-sensor-de-temperatura-

DS18B20.html. [Accessed: 14-Dec-2020].

[12] "Sensor de temperatura DS18B20 con protector metálico - Sumador." [Online]. Available:

https://sumador.com/en/products/sensor-detemperatura-ds18b20-con-protector. [Accessed: 14-Dec-2020].

[13] "Tutorial módulo sensor de luz BH1750." [Online].

Available: https://www.naylampmechatronics.com/blog/44 _Tutorial-módulo-sensor-de-luz-BH1750.html. [Accessed: 14-Dec-2020]. 